Onkologie 1997;20:18186-188

\title{
Contents - Inhalt, Vol. 20, No. 3, 1997
}

\section{Review Articles}

Übersichtsarbeiten

190 Positron Emission Tomography for Diagnosis of Breast Tumors

Dose, J. (Hamburg); Avril, N; Graeff, H. (München); Jänicke, F, (Hamburg)

196 High-Dose Chemotherapy with Autologous

Haematopoietic Stem Cell Support in Advanced Ovarian Cancer

Möbus, V; von Schilling, C; Kreienberg, R.; Frickhofen, N.; Herrmann, F. (Ulm)

204 Ibandronate in Malignant Bone Diseases and Osteoporosis - Preclinical Results

Bauss, F. (Mannheim)

210 Psychological Interventions in Patients with Gynecological Cancer

Jürgensen, O. (Frankfurt/M.); Neises, M. (Mannheim)

190 Die Positronenemissionstomographie in der Diagnostik von Mammatumoren

Dose, J. (Hamburg); Avril, N.; Graeff, H. (München); Jänicke, F. (Hamburg)

196 Hochdosis-Chemotherapie mit autologem hämatopoetischem Stammzellersatz bei fortgeschrittenem Ovarialkarzinom

Möbus, V.; von Schilling, G; Kreienberg, R.; Frickhofen, N.; Herrmann, F. (Ulm)

204 Ibandronat bei malignen Knochenerkrankungen und Osteoporose - Präklinische Ergebnisse

Bauss, F. (Mannheim)

210 Möglichkeiten psychologischer Intervention bei Patientinnen mit gynäkologischen

Karzinomen

Jürgensen, O. (Frankfurt/M.); Neises, M. (Mannheim)

Original Articles

Originalarbeiten

216 Survival Rates and Prognosis Factors of Endometrial Cancer Stage I

Osmers, R.G.W.; Niemeyer, E. A.; Kron, M.; Kuhn, W. (Göttingen)

216 Überlebensraten und Prognosefaktoren beim Endometriumkarzinom Stadium I

Osmers, R.G.W.; Niemeyer, E. A.; Kron, M.; Kuhn, W. (Göttingen)

222 Intravenous Weekly High-Dose Infusion of

5-Fhlorouracil and Folinic Acid in Pretreated Patients with Metastatic Colorectal Cancer

Lorenz, M.; Staib-Sebler, E.; Gog, G; Petrowsky, H; Encke, A. (Frankfurt/M.)

226 PET Studies of Perfusion and Glucose Metabolism in Patients with Untreated Head and

Neck Tumours

Kuhn, G.-D.; Reißer, G; Dimitrakopoulou-Strauss, A.; Oberdorfer, F.; Strauss, L. G.

(Heidelberg) 
222 Systemische wöchentliche Hochdosis-Chemotherapie mit 5-Fluorouracil und Folinsäure bei vorbehandelten Patienten mit metastasierenden kolorektalen Karzinomen

Lorenz, M,; Staib-Sebler, E.; Gog, G; Petrowsky, H.; Encke, A. (Frankfurt/M.)

226 PET-Studien der Perfusion und des Glukosestoff-wechsels bei Patienten mit unbehandelten Kopf-Hals-Tumoren

Kuhn, G.-D.; Reißer, C; Dimitrakopoulou-Strauss, A.; Oberdorfer, F; Strauss, L. G. (Heidelberg)

Ongoing Clinical Trials

231 Adjuvant Radio-Chemotherapy with 5-Fluorouracil and Folinic Acid in Stage II and III Rectal Cancer: Interim Analysis

Hartung, G; Queißer, W,; Maas, K; Diezler, P.; Hagmüller, E. (Mannheim); Edler, L,; KoppSchneider, A. (Heidelberg); Weniger, I; Baur, A.; Pabst, T. (Erfurt); Jacob, I.; Wojatschek, C. (Ludwigshafen); Seifert, A. (Hamburg); Weh, H.-X; Janssen, N. (Siegen); Engel, M.

(Mönchengladbach und Darmstadt); Drost, H; Stockert, H. (Hagen und Mannheim); Lehnigk, S. (Zeitz) for the study group Colon and Rectum Carcinoma, Mannheim

Laufende klinische Studien

231 Adjuvante Radio-Chemotherapie mit 5-Fluorouracil und Folinsäure bei Rektumkarzinomen im Stadium II und III: Zwischenauswertung

Hartung, G; Queißer, W,; Maas, K; Diezler, P.; Hagmüller, E. (Mannheim); Edler, L.; KoppSchneider, A. (Heidelberg); Weniger, J,; Baur, A.; Pabst, T. (Erfurt); Jacob, I.; Wojatschek, C. (Ludwigshafen); Seifert, A. (Hamburg); Weh, H.-J.; Janssen, N. (Siegen); Engel, M.

(Mönchengladbach und Darmstadt); Drost, H; Stockert, H. (Hagen und Mannheim); Lehnigk, S. (Zeitz) für die Study Group Colon and Rectum Carcinoma, Mannheim

Continued on page 188

Fortsetzung aufSeite 188

Bibliographischer Hinweis: Inhaltsverzeichnisse dieser Zeitschrift erscheinen regelmäßig in current contents ${ }^{\circledR}$ sowie in anderen bibliographischen Diensten.

Contents $\boldsymbol{\text { Inhalt }}$

OHKOLOGIE

Vol. 20, Issue 3, June 1997

Vol. 20, Heft 3, Juni 1997

235 Acceptance and Toxicity of Postoperative Adjuvant Therapy in Colon and Rectal Cancers: Interim Report on the Trials FOGT1 and FOGT2 of the German 'Forschungsgruppe Onkologie Gastrointestinaler Tumoren (FOGT)'

Link, K.H.; Staib, L.; Bernhart, H. (Ulm); Kreuser, E.D. (Berlin); Suhr, P.; Röttinger, E.; Beger, H.G. (Ulm) for the 'Forschungsgruppe Onkologie Gastrointestinaler Tumoren (FOGT)' 235 Akzeptanz und Toxizitat der postoperativen adjuvanten Therapie bei Kolon- und Rektumkarzinomen: Zwischenbericht der FOGT1- und FOGT2-Studien der «Forschungsgruppe Onkologie Gastrointestinaler Tumoren (FOGT)»

Link, K.H.; Staib, L.; Bernhart, H. (Ulm); Kreuser, E.D. (Berlin); Suhr, P.; Röttinger, E.; Beger, H.G. (Ulm) für die «Forschungsgruppe Onkologie Gastrointestinaler Tumoren (FOGT)»

Oncological Education

239 Apoptosis in the Immune System: Murder or Suicide 
Krammer, P. H. (Heidelberg)

Onkologische Fortbildung 239 Apoptose im Immunsystem: Mord oder Selbstmord

Krammer, P. H. (Heidelberg)

244 ESMO Certification and ESMO-MORA: $\quad 244$ ESMO-Zertifizierung und ESMOMORA: Ein Beitrag

A Contribution to Quality Protection in Medical Tumor zur Qualitätssicherung bei der medikamentösen

Therapy Tumortherapie

Illiger, H. J. (Oldenburg) Illiger, H. J. (Oldenburg)

Clinical Information

248 Status of Oncology in Germany - Surgical Field

Pichlmaier, $\mathrm{H}_{2}$; Türler, A. (Köln)

252 The Hospital: An Incurable Patient?

Oberender, P. (Bayreuth)

256 Chronic Lymphatic Leukemia and Hairy-Cell Leukemia. Diagnostics and Therapy

Konsensustagung, Berlin, February 1996

261 Evaluation of Quality of Life in Patients with Head and Neck Cancer

Bergler, W.; Juncker, C; Hörmann, K. (Mannheim)

Klinische Informationen 248 Stellung der Onkologie in Deutschland - operatives Fach

Pichlmaier, H; Türler, A. (Köln)

252 Das Krankenhaus: Ein unheilbarer Patient?

Oberender, P. (Bayreuth)

256 Chronische lymphatische Leukämie und Haarzellen-Leukämie. Diagnostik und Therapie Konsensustagung, Berlin, Februar 1996

261 Die Messung der Lebensqualität bei Patienten mit Kopf-Hals-Tumoren

Bergler, W.; Juncker, G; Hörmann, K. (Mannheim)

266 Book Reviews

266 Buchbesprechungen

270 Industrial Forum

270 Industrieforum

272 Reports of Oncological Societies

272 Mitteilungen onkologischer Gesellschaften

185 Imprint

185 Impressum

188 